\title{
REVIEW \\ Solving difficult hepatobiliary problems in children
}

\author{
A J W Millar
}

Most difficult hepatobiliary (HPB) problems in infancy and childhood result from pathological anatomical/mechanical derangements; therefore, surgery on the liver and bile ducts depends on a detailed understanding of liver structure, function and repair response to injury or disease. The surgeon must be aware of the very diverse range of anatomical variations. Perhaps key to improving the outcome of paediatric HPB surgery is centralised management and associating this with a paediatric liver transplant programme, which adds expertise and, frequently, the added benefit of adult HPB surgical input to paediatric surgical care. In the United Kingdom, this has resulted in excellent measurable benefit, particularly in the management of biliary atresia, but also of choledochal cysts, portal hypertension and liver tumours. These conditions are briefly discussed here, with focus on the technical aspects of operative management.

S Afr Med J 2012;102(11):872-875. DOI:10.7196/SAMJ.6135
Most difficult hepatobiliary (HPB) problems in infancy and childhood result from pathological anatomical/mechanical derangements; therefore, surgery on the liver and bile ducts depends on a detailed understanding of liver structure, function and repair response to injury or disease. ${ }^{1}$ Most importantly, the surgeon should be aware of the very diverse range of anatomical variations. Perhaps key to improved outcomes in paediatric HPB surgery is centralised management and associating this with a paediatric liver transplant programme, which adds expertise and, frequently, the added benefit of adult HPB surgical input to paediatric surgical care. This has been performed in the United Kingdom (UK) with excellent measurable benefit. ${ }^{2,3}$

\section{Biliary atresia \\ Portoenterostomy}

The surgery for biliary atresia (BA), initially proposed by Kasai in 1959, has seen little change, although numerous modifications have been proposed. ${ }^{4}$ There is now remarkable consensus over most aspects of the surgery, but less in the role of the use of steroids, prophylactic antibiotics, choleretic agents and the prevention and treatment of cholangitis. With a combination of early surgery, pre-surgery gut sterilisation, minimally invasive open surgery (not laparoscopy), a high starting dose, short course and early taper steroid protocols, antibiotic prophylaxis and choleretics (ursodeoxycholic acid, cholestyramine and phenobarbitone), $>80 \%$ sustained clearance of jaundice has been achieved. ${ }^{5}$ This has led to a reduced number of infants and children

\footnotetext{
Alastair J W Millar currently holds the Charles F M Saint Chair of Paediatric Surgery at the University of Cape Town and is Head of Surgery at the Red Cross War Memorial Children's Hospital (RCWMCH), Cape Town. He has a special interest in paediatric surgical liver disease. He was instrumental in initiating the liver transplant programme at the RCWMCH after undertaking a fellowship in the University Department of Surgery, Addenbrooke's Hospital, Cambridge, under Professor Sir Roy Calne in 1990, and was Clinical Lead in Hepatopancreaticobiliary Surgery and Transplantation at the Birmingham Children's Hospital NHS Trust (2004 - 2007).
}

Corresponding author: A J W Millar (alastair.millar@uct.ac.za) requiring liver transplantation during the childhood years. Although this may only delay the need for liver transplantation (about $85 \%$ of all BA cases eventually require transplantation even after a successful Kasai portoenterostomy), a childhood complicated by the need for immunosuppression can be avoided.

The biliary tree should be approached via a right-sided transverse or oblique subcostal incision, which can be extended across the midline. A laparoscopic Kasai procedure has a poorer long-term outcome and is currently not recommended. ${ }^{6}$ If a diagnosis has not been established pre-operatively, then an operative cholangiogram should be performed via a catheter in the gallbladder. If there is bile in a normal-looking gallbladder, a diagnosis of BA can be excluded, provided that patency of the common bile duct to the duodenum is shown. A gallbladder filled with clear mucus suggests type $2 \mathrm{BA}$ and a cholangiogram may show a thinly patent bile duct but absent proximal ducts. At this stage, only complete visualisation of the whole biliary tree can exclude BA. The hepatic suspensory ligaments may be divided and the liver rotated anteriorly to expose its inferior surface in the incision. It is rarely necessary to 'deliver' the liver through the incision. Although this manoeuvre provides good exposure to the porta hepatis, possible adverse effects include congestion of the liver and, on occasion, acute impairment of venous return and cardiac asystole. A folded swab can be placed posterior to the right lobe to lift the liver forward. The gallbladder remnant should be dissected off the liver and the obliterated common biliary tract divided distally. Using the gallbladder remnant for gentle traction, the residual fibrotic bile duct should be elevated to separate it from the underlying portal vein and adjacent hepatic artery and then dissected free from the right and left branches of the portal vein. There is debate as to how far laterally the dissection should be extended. It is usually sufficient to stop where the hepatic artery enters the liver parenchyma. Small portal venous radicles entering the caudate lobe must be ligated and divided with fine sutures to expose the posterior aspect of the portal plate. After the portal plate has been fully dissected, a $35-40 \mathrm{~cm}$ long Roux limb of jejunum, brought up through the mesentery of the transverse colon, must be created. The proximal transection of jejunum should be close (approximately $10 \mathrm{~cm}$ ) to the duodenojejenal (DJ) flexure. The open end should be sutured or ligated closed and inverted with a purse-string suture. The blood supply and venous drainage from the Roux limb should be fully preserved. The proximal end of the Roux must be incised and tailored to the size of the portal plate. The fibrotic portal plate must now be transected high at the level of the porta hepatis without entering the liver parenchyma. This 
is best performed using super-cut curved scissors, commencing on the left lateral aspect. Bleeding from the cut surface of the portal plate should be minimal. Traction sutures placed in the caudate lobe may facilitate exposure. A complete resection of the extrahepatic biliary tree is advisable in all types of BA, except for the uncommon case in which there is a significant remnant of patent bile duct in the porta hepatis. Biliary continuity should be restored using the Roux limb, which is anastomosed to the transected tissue in the porta hepatis as a portoenterostomy with continuous or interrupted 5/0 absorbable sutures. The posterior layer of sutures should be placed well clear of the transected portal plate and the lateral and anterior layer should be placed superficially in Glisson's capsule. The operation must be completed by ensuring that the defects in the mesentery are sutured closed and the Roux limb lies appropriately without twisting. Various types of valves have been advocated (mucosal flap and intussusception valves), but there is no strong evidence for their efficacy. ${ }^{7} \mathrm{~A}$ drain is unnecessary. In some BA cases, operative cholangiography may show a patent lower common bile duct in continuity with the gallbladder, with the atretic process restricted to the common hepatic and hepatic ducts. Reconstruction of the biliary tract using the gallbladder (portocholecystostomy) after resection of the remnants of the bile ducts has been suggested to be effective in preventing post-operative cholangitis. However, obstructive post-operative complications are frequent with this technique and it has largely been abandoned.

\section{Post-operative care and long-term complications}

Antibiotics are administered intravenously (IV) in the immediate post-operative period and replaced by oral antibiotic prophylaxis after the return of bowel activity. This can be continued for the first year according to protocol. Choleretics (cholestyramine, ursodeoxycholic acid $(10 \mathrm{mg} / \mathrm{kg} /$ dose twice daily) and phenobarbitone $(5 \mathrm{mg} / \mathrm{kg} /$ dose nocte)) and vitamins $\mathrm{A}, \mathrm{D}, \mathrm{E}$, and $\mathrm{K}$ are also prescribed for at least 1 year. Ranitidine ( $1 \mathrm{mg} / \mathrm{kg} /$ dose 3 times daily) is administered for gastric protection for the duration of the course of steroids. Steroids have been recommended on the basis that they may reduce scar tissue formation and improve bile flow after portoenterostomy; no controlled trials have confirmed or refuted their efficacy, although observational evidence supports their use. Dose, time of treatment initiation and duration vary widely; prospective trials are in progress to define best practice. ${ }^{8}$ The best available evidence suggests that a short course of initial high-dose steroid ( $4-5 \mathrm{mg} / \mathrm{kg} /$ day) with rapid tapering is the most effective:

- Steroids(anoptional2-weekcourse):e.g. $20 \mathrm{mgmethylprednisolone}$

(IV) on day 1 , decreasing to $2.5-5 \mathrm{mg} /$ day, followed by $5 \mathrm{mg}$ prednisolone (oral) daily for 1 week

- Antibiotics (IV) for 5 days: gentamicin $(2.5 \mathrm{mg} / \mathrm{kg} /$ dose $) 3$ times daily (levels needed) and amoxicillin $(25 \mathrm{mg} / \mathrm{kg} /$ dose $) 3$ times daily

- Antibiotic prophylaxis (started on post-operative day 6): cephalexin $(12.5 \mathrm{mg} / \mathrm{kg} /$ dose $)$ twice daily for 1 month, or ciprofloxacin $(5-10 \mathrm{mg} / \mathrm{kg} / \mathrm{dose})$ twice daily (oral) with extended prophylaxis (od) dose.

Complications of portoenterostomy include: ascending bacterial cholangitis, cirrhosis, portal hypertension, metabolic and nutritional consequences of cholestasis, intrahepatic cyst formation, hepatopulmonary syndrome, pulmonary hypertension, and malignant change in the liver (rare).

\section{Ascending bacterial cholangitis}

This serious complication is most common in the first year following portoenterostomy. Episodes of infection occur in approximately 40 - $50 \%$ of the infants, most commonly in those who have achieved at least some degree of bile flow. The complication, characterised by worsening jaundice, fever and acholic stools, is diagnosed by blood culture, percutaneous liver biopsy or aspiration blood culture. A wide range of causative organisms may be identified, including Escherichia coli, Proteus and Klebsiella species, but suspected cases must be treated early and empirically with broadspectrum antibiotics (e.g. ceftazidime, amoxicillin, ciprofloxacin and gentamicin or piperacillin and amikacin), before the detailed results of investigations are available.

Several operative modifications have been made to Kasai's original portoenterostomy to reduce the incidence of cholangitis, including diversion stomas and the formation of anti-reflux valves in the limb of the Roux loop. Despite the theoretical benefits of such modifications, in practice they confer little additional benefit, and equally good results are obtained from the use of a long Roux loop.

Cholangitis may occur some years after portoenterostomy in children with otherwise good liver function. In such cases, partial obstruction of the Roux loop, perhaps secondary to an adhesion or twist in the loop, must be excluded, as this can be relieved by surgery. Percutaneous trans-hepatic cholangiography (PTC) and radionuclide hepatic imaging are essential to identifying the site of the obstruction in these cases. Prolonged antibiotic prophylaxis may be unnecessary, particularly if there is no obstruction of the Roux loop. If cholangitis recurs frequently despite these measures and with deteriorating liver function, then liver transplantation should be considered.

\section{Results}

Several variables have been studied to predict the effectiveness of portoenterostomy; some derived from peri-operative data, e.g. age at surgery, macroscopic appearance of the bile ducts, microscopic analysis of the resected specimen, and liver histopathology., ${ }^{2,9}$ The extent of histological abnormality (degree of fibrosis) at the time of surgery may indicate a poorer prognosis, but this finding has not been consistent. The degree of portal hypertension at the time of the procedure is correlated with a shorter time to requiring liver transplantation, reflecting liver pathophysiology in a more functional way. ${ }^{10}$ Although disputed, the surgeon's experience has also been implicated as an important prognostic factor. ${ }^{11}$ In a personal series of patients operated on at a mean age of 52 days over a 3.5-year period (2004 - 2007), clearance of jaundice was achieved in 23/29 cases (79\%). Perhaps more importantly, improved outcome has been associated with greater centre caseload (and consequently, greater experience), and better communication between major centres and peripheral units. ${ }^{12}$ The age at which surgery is performed is the single most widely quoted prognostic variable, although some have shown little relationship in infants aged $<10$ weeks. ${ }^{13,14}$ However, in infants older than 100 days at the time of portoenterostomy, uncorrected atresia of the bile ducts results in progressive intrahepatic disease and a clear detrimental effect of age on survival has been demonstrated. In summary, the post-operative volume of bile flow is probably related to the size of bile ductules at the porta hepatis, while the longterm quality of survival in those with adequate bile flow depends on the severity of secondary liver damage at the time of surgery and the incidence and severity of post-operative cholangitis. ${ }^{13,15,16}$

Approximately $70-80 \%$ of infants show evidence of bile flow after surgery, which is adequate to ensure survival to 5 years of age in $>65 \%$ of cases, reducing the need for paediatric liver transplantation. Furthermore, series from Japan, France, the USA and the UK suggest that $30-40 \%$ of paediatric patients survive to 10 years of age with their native liver intact following portoenterostomy, although approximately $40 \%$ have abnormal results on liver function tests. In a French series, 23\% (63/212) of patients who underwent 
portoenterostomy between 1968 and 1983 were alive with their native liver intact 20 years after surgery, although all but 2 had signs of cirrhosis. ${ }^{17}$

\section{Choledochal cysts}

Total cyst excision has been the treatment of choice for decades. A cholangiogram is performed if the anatomy of the cyst has not been defined pre-operatively with ultrasound, computed tomography or magnetic resonance cholangiopancreatography. A sample of bile should be taken for amylase, lipase and culture testing. The extrahepatic cyst must be excised completely, keeping to the relatively avascular plane just deep to the peritoneum. Distal excision should be complete down to the level of the pancreatic duct junction, which can be confirmed endoscopically using a choledochoscope or, if not available, a paediatric cystoscope in the case of fusiform cysts with pancreaticobiliary malunion. A vessel loop passed around the cyst is useful to lift it clear of the portal vein. The proximal anastomosis should ideally be at the level of the right and left hepatic duct confluence, without leaving a cuff of residual cyst. The cyst should not be excised too high in the porta hepatis, as it is easy to cut off the cyst above the entrance of the right and left hepatic ducts into the cyst. Extending the width of the anastomosis by incising along the left hepatic duct, which usually lies outside of the liver, avoids stenosis. ${ }^{18}$ Roux-en-Y biliary enteric drainage is the preferred route, as long-term bile reflux gastritis occurs in up to $25 \%$ of cases where a hepatico-duodenostomy has been performed. Minimally invasive techniques are popular in Asia where choledochal cysts are more commonly seen; the simpler hepatico-duodenostomy procedure appears to be favoured. ${ }^{19,20}$

\section{Foregut atresia and biliary anomalies}

These anomalies, noted with increasing frequency, include the hepatocystic duct where the common hepatic or right hepatic duct inserts into the gallbladder, choledochal cysts, stenosis and choledochocoele. ${ }^{21}$

\section{Portal hypertension}

The meso-Rex shunt, first described by de Ville de Goyet ${ }^{22}$ using an autologous internal jugular vein graft, is now the preferred method of treatment for portal cavernoma. ${ }^{23}$ There have been several further technical innovations including use of the coronary vein, inferior mesenteric vein and spliced saphenous veins when the jugular vein is not available. Selective shunts are preferred if the Rex vein is not patent (30\% of cases) and oesophageal varices are not controlled by banding or sclerotherapy. Outcomes are excellent, with long-term vein patency in nearly all cases, and good evidence of restoration of hepatic nutrition, with increased liver growth, resolution of the cavernoma and biliopathy, a reduction in spleen size, and an added bonus of improved intellectual performance. ${ }^{24,25}$

\section{Budd-Chiari syndrome}

This rare but devastating disease, which may be associated with a thrombophilic diathesis or anatomical web, can be managed in the early stages with trans-jugular stenting. The Senning hepato-atrial patch operation is less commonly used, but can be successful. If liver cirrhosis is established, then transplantation is indicated. ${ }^{26-28}$

\section{Hepatic vascular tumours and malformations}

Glucose transporter-1 (GLUT-1)-negative infantile haemangiomas may not respond to propranolol and steroids. If the infant is small and unstable due to cardiac failure, thrombocytopenia and/or jaundice, then hepatic artery ligation may be a simpler, effective and indeed less invasive intervention than embolisation. Laparoscopic occlusion of the main feeding arteries is also effective. Transplantation has been used as a last resort in a few cases. ${ }^{29}$

\section{Tumour surgery}

Extending the limits of hepatic resection has become a fine art. ${ }^{30-32}$ Perhaps in some cases this is a bridge too far, as ill-advised and poorly conducted extended hepatic resections may lead to early post-operative liver failure and/or the necessity for emergency liver transplantation. Also, transplantation after local recurrence following resection has a poorer outcome. Good surgical exposure is achieved by a large subcostal incision, which can be extended to the xiphisternum in the midline, and by using one of the selfretaining retractor systems. Intra-operative ultrasound can define the exact relationship between the area to be resected and the major venous anatomy. Obstructed and dilated bile ducts can be cut flush with the residual cut surface of the liver and drained with a Rouxen-Y hepaticojejunostomy. ${ }^{33}$ If the extrahepatic bile ducts are to be preserved, it is essential to ensure that arterial supply has not been compromised. Inflow occlusion (Pringle) is routine and it is wise to have control of the inferior vena cava above and below the liver prior to commencing resection. Occasionally, total vascular exclusion is useful to control bleeding. Many technical aids are available for parenchymal transaction, but personal preference is for non-stick bipolar diathermy and titanium haemostatic clips. Topical haemostatic agents can help with ooze, and the Argon beam coagulator helps with haemostasis over a large cut section, but the Argon is a 'luxury' in my opinion. A vascular stapler for the hepatic veins is particularly useful in larger children. The key to successful resection is to mobilise the liver fully off the retrohepatic inferior vena cava and subsequently define and control the vascular inflow and the hepatic venous outflow to the sector(s) to be resected. Great care should be taken to ligate or clip the venous connections from segments 8 and 1 to avoid bleeding from the cava or under-surface of the liver. Low central venous pressure helps to minimise blood loss. Further 'tips and tricks': (i) taking too much liver and leaving a remnant at risk for 'small-for-size' syndrome can be avoided by pre-operative portal vein embolisation/ligation, allowing for growth of the proposed liver remnant prior to resection; (ii) avoid hepatic congestion by ensuring that there is no hepatic venous outflow obstruction from torsion/kinking of the residual liver remnant; and (iii) reduce inflow perfusion with somatostatin infusion, splenic artery ligation and even temporary partial porto-systemic shunting. In the post-operative period, management includes medical support for the liver with infusion of fresh-frozen plasma to maintain haemostasis and protein $\mathrm{C}$ levels and $\mathrm{N}$-acetyl cysteine infusion as prophylaxis against metabolic liver failure. Where the hepatic veins are involved by tumour, a 'proximal' hepatectomy can be performed safely if sufficiently large accessory right hepatic Baer's vein(s) are draining the residual liver into the inferior vena cava. ${ }^{34}$ Ex vivo perfusion and bench surgery have also been performed successfully for the rare benign tumour involving the cavo-hepatic confluence.

1. Baumann U, Brown RM. Structure function and repair of the liver in diseases of the liver and biliary system in children. United Kingdom: Wiley-Blackwell, 2008. [http://dx.doi. org/10.1002/9781444300536.ch1

2. Davenport M, Ong E, Sharif K, et al. Biliary atresia in England and Wales: Results of centralization and new benchmark. J Pediatr Surg 2011;46(9):1689-1694. [http://dx.doi.org/10.1016/j. jpedsurg.2011.04.013]

3cKiernan PJ, Baker AJ, Kelly DA. The frequency and outcome of biliary atresia in the UK and Ireland. Lancet 2000;355(9197):25-29.

4. Kasai M. Treatment of biliary atresia with special reference to hepatic porto-enterostomy and its modifications. Prog Pediatr Surg 1974;6:5-52. 


\section{FORUM}

5. Meyers RL, Book LS, O'Gorman MA, et al. High-dose steroids, ursodeoxycholic acid, and chronic intravenous antibiotics improve bile flow after Kasai procedure in infants with biliary atresia. J Pedia Surg 2003,38(3).406-411. [http.//dx.doi.org/10.1053/psu.2003.50069]

6. Ure BM, Kuebler JF, Schukfeh N, Engelmann C, Dingemann J, Petersen C. Survival with the native liver after laparoscopic versus conventional kasai portoenterostomy in infants with biliary atresia: $\mathrm{A}$

7. Saekpi M, Nakamo M, Hagane K, Shimizu K. Effectiveness of an intussusceptive antireflux valve to preven
. ascending cholangitis after hepatic portojejunostomy in biliary atresia. J Pediatr Surg 1991;26(7):800-803 8. Muraji T, Nio M, Ohhama Y, et al. Postoperative corticosteroid therapy for bile drainage in biliar atresia - a nationwide survey. J Pediatr Surg 2004;39(12):1803-1805. [http://dx.doi.org/10.1016/ jpedsurg.2004.08.019

9. Howard ER, MacLean G, Nio M, Donaldson N, Singer J, Ohi R. Survival patterns in biliary atresia an comparison of quality of life of long-term survivors in Japan and England. J Pediatr Surg 2001;36(6):892897. [http://dx.doi.org/10.1053/jpsu.2001.23965]

10. Duché M, Fabre M, Kretzschmar B, Serinet MO, Gauthier F, Chardot C. Prognostic value of portal pressure at the time of Kasai operation in patients with biliary atresia. J Pediatr Gastroenterol Nut 2006;43(5):640-645. [http://dx.doi.org/10.1097/01.mpg.0000235754.14488.f9]

11. Lloyd D, Jones M, Dalzell M. Surgery for biliary atresia. Lancet 2000;355(9209):1099-1100. [http://dx.do org/10.1016/S0140-6736(05)72221-2]

2. Serinet MO, Broué $\mathrm{P}$, Jacquemin E, et al. Management of patients with biliary atresia in France: results of a decentralized policy 1986 - 2002. Hepatology 2006;44(1):75-84. [http://dx.doi.org/10.1002/hep.21219] 3. Tagge DU, Tagge EP, Drongowski RA, Oldham KT, Coran AG. A long-term experience with biliary atresia. Reassessment of prognostic factors. Ann Surg 1991;214(5):590-598.

4. Haas JE. Bile duct and liver pathology in biliary atresia. World J Surg 1978;2(5):561-569. [http://dx.doi. org $/ 10.1007 / \mathrm{BF} 01556047$

15. Davenport M, Kerkar N, Mieli-Vergani G, Mowat AP, Howard ER. Biliary atresia: the King's College Hospital experience (1974 - 1995). J Pediatr Surg 1997;32(3):479-485. [http://dx.doi.org/10.1016/S00223468(97)90611-4]

16. Shneider BL, Brown MB, Haber B, et al. A multicenter study of the outcome of biliary atresia in the United States, 1997 to 2000. J Pediatr 2006;148(4):467-474. [http://dx.doi.org/10.1016/S00223468(97)90611-4]

17. Lykavieris P, Chardot C, Sokhn M, Gauthier F, Valayer J, Bernard O. Outcome in adulthood of biliary atresia: a study of 63 patients who survived for over 20 years with their native liver. Hepatology 2005;41(2):366-371. [http://dx.doi.org/10.1002/hep.20547]

8. Stringer MD. Wide hilar hepaticojejunostomy: the optimum method of reconstruction after choledochal cyst excision. Pediatr Surg Int 2007;23(6):529-532.

19. Miyano T, Yamataka A, Kato Y, et al. Hepaticoenterostomy after excision of choledochal cyst in children a 30-year experience with 180 cases. J Pediatr Surg 1996;31(10):1417-1421. [http://dx.doi.org/10.1016/ S0022-3468(96)90843-X]

20. Miyano T, Yamataka A, Kato Y, et al. Laparoscopic-assisted total cyst excision of choledochal cyst and Roux-en-Y hepatoenterostomy. J Pediatr Surg 2004;39(11):1663-1666. [http://dx.doi.org/10.1016/]. jpedsurg.2004.07.012
21. Mali V, Wagener S, Sharif K, Millar AJ. Foregut atresias and bile duct anomalies: rare, infrequent o common?! Pediatr Surg Int 2007;23(9):889-895. [http://dx.doi.org/10.1007/s00383-007-1921-y]

22. De Ville de Goyet J, Alberti D, Clapuyt P, et al. Direct bypassing of extrahepatic portal venou obstruction in children: a new technique for combined hepatic portal revascularization and treatment

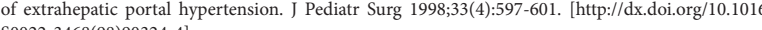

23. Superina R, Shneider B, Emre S, Sarin S, de Ville de Goyet J. Surgical guidelines for the management of extra-hepatic portal vein obstruction. Pediatr Transplant 2006;10(8):908-913.

4. Sharif K, McKiernan P, de Ville de Goyet J. Mesoportal bypass for extrahepatic portal vein obstructio in children: Close to a cure for most! J Pediatr Surg 2010;45(1):272-276. [http://dx.doi.org/10.1016/] jpedsurg.2009.08.019

25. Mack CL, Zelko FA, Lokar J, et al. Surgically restoring portal blood flow to the liver in children with primary extrahepatic portal vein thrombosis improves fluid neurocognitive ability. Pediatric 2006;117(3):e405-e412. [http://dx.doi.org/10.1542/peds.2005-1177]

26. Cauchi JA, Oliff S, Baumann U, et al. The Budd-Chiari syndrome in children: the spectrum of management.J Pediatr Surg 2006;41(11):1919-1923. [http://dx.doi.org/10.1016/j.jpedsurg.2006.06.045] 27. Odell JA, Rode H, Millar AJ, Hoffman HD. Surgical repair in children with the Budd-Chiari syndrom J Thorac Cardiovasc Surg 1995;110:916-923. [http://dx.doi.org/10.1016/S0022-5223(05)80158-8]

28. Rerksuppaphol S, Hardikar W, Smith AL, et al. Successful stenting for Budd-Chiari syndrom after pediatric liver transplantation: a case series and review of the literature. Pediatr Surg In 2004;20(2):87-90.

29. Azizkhan R. Chapter 97: Hemangiomas. In: Gupta DK, Sharma S, Azizkhan ED, eds. Pediatric Surgery Diagnosis and Management. Volume 2. New Delhi, India: Jaypee Brothers, 2009:1079-1098.

30. Morland B, de Ville de Goyet J. Primary Hepatic Tumours. In: Kelly D, ed. Diseases of the Liver an Biliary System in Children. United Kingdom: Wiley-Blackwell, 2008:475-500.

11. Stringer M. Liver Resections and Allograft Preparation. In: Gupta DK, Sharma S, Azizkhan ED, eds. Pediatric Surgery Diagnosis and Management. New Delhi, India: Jaypee Brothers 2009:948-968.

32. Clavien PA, Petrowsky H, DeOliveira ML, Graf R. Strategies for safer liver surgery and partial liver transplantation. N Engl J Med 2007;356(15):1545-1559. [http://dx.doi.org/10.1056/NEJMra065156]

33. Millar AJ, Hartley P, Khan D, Spearman W, Andronikou S, Rode H. Extended hepatic resection with transplantation back-up for an "unresectable" tumour. Pediatr Surg Int 2001;17(5-6):378-381. [http:/ dx.doi.org/10.1056/NEJMra065156]

34. Baer HU, Dennison AR, Maddem GJ, Blumgart LH. Subtotal hepatectomy: A new procedure based on the inferior right hepatic vein. Br J Surg 1991;78(10):1221-1222. [http://dx.doi.org/10.1002/ bjs.1800781024

Accepted 26 July 2012 